\title{
Ein grosser Schritt für die Rehabilitation
}

\section{Daniel R. Perez}

PD Dr. med., Universitätsklinikum Hamburg-Eppendorf

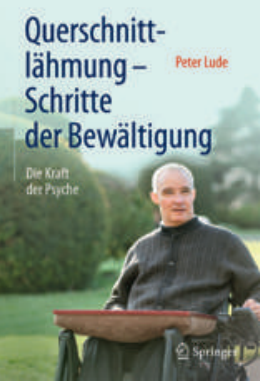

Peter Lude

Querschnitt-

lähmung -

Schritte der

Bewältigung

Berlin, Heidelberg:

Springer-Verlag;

2016.

297 Seiten. $26.80 \mathrm{CHF}$

ISBN

978-3-662-47969-8
Dieses Buch von Dr. Peter Lude eröffnet einen ganz neuen Blick auf das Leben, von einer ganz anderen Warte aus, als man üblicherweise so zu lesen bekommt. Es zeigt, wo die Essenz des Lebens sich befindet. Das liegt vor allem daran, dass es (auch) eine persönliche, sehr spannend und berührend geschriebene Lebensgeschichte ist. Eingebettet in diese Lebensgeschichte steckt natürlich auch fachlich eine Menge Schubkraft darin, ein solides Faktenwissen. Das ist eine sehr gelungene Kombination.

Dadurch, dass das physische Leben als Tetraplegiker stark eingeschränkt ist, wird klar, dass die Essenz des Lebens, die Lebensquelle, nicht in der Bewegung, der äusserlichen Aktion, sondern im Denken, in der Seele oder eben in der Psyche zu finden ist.

Ich finde den Ansatz entscheidend, dass die Psyche schon von Anfang an nach Eintritt der Querschnittlähmung gezielt gestärkt wird und das Leiden aller Beteiligten, auch der Angehörigen, vollgültig ins Leben integriert wird, um Bewältigung möglich zu machen, - und nicht auf die StandardDepression (zum Beispiel als Teil der 4-stufigen Trauerbewältigung nach Schema F) gesetzt wird, ohne die eine «realistische» Einstellung, die ja das Mass aller Dinge sein soll, nicht möglich sei.

\section{Ich finde den Ansatz entscheidend, dass die Psyche schon von Anfang an nach Eintritt der Querschnittlähmung gezielt gestärkt wird.}

In seinen Forschungsarbeiten hat Dr. Lude, Fachpsychologe für Psychotherapie FSP, Dozent für Rehabilitationspsychologie und zugleich ein international ausgewiesener Rehabilitationsexperte, überzeugend gezeigt, dass bei Patienten nach schwerer Wirbelsäulenverletzung unmittelbar natürliche, starke lebenszuwendende psychische Reaktionen einsetzen. Diese von aussen nicht sichtbaren Reaktionen sollten künftig vom Behandlungsteam erkannt und unterstützt und nicht medikamentös oder therapeutisch (weg-)behandelt werden. Als ehemaliger, vollständig wiederhergestellter Unfallpatient und in meiner heutigen klini- schen Tätigkeit als Chirurg scheint mir das Konzept des psychischen "Airbag-Effekts» ein neues Verständnis der psychologischen Traumaverarbeitung zu bieten, das insbesondere auch für die langfristige Anpassung an durch Unfall oder Krankheit veränderte Lebensumstände von grossem Nutzen ist. Nachträglich meine persönliche Erfahrung als Rehabilitand betrachtend, kann ich heute sagen, dass Patienten solche wie von Dr. Lude beschriebenen Reaktionen und Prozesse erleben, aber man diese bisher nicht auf eine konstruktive Weise verstanden und keine adäquate Ausdrucksweise dafür gefunden hatte. Aufgrund meiner zahlreichen und vielfältigen Patientenbehandlungen kann ich feststellen, dass die beschriebenen Phänomene gut nachvollziehbar sind und durchaus Allgemeingültigkeit aufweisen.

Das Buch sollte von allen gelesen werden, die mit Rehabilitation zu tun haben.

Wie gehen die Reha-Leute mit dem Kontrast zwischen ihren Fragebögen, endlosen Tests und Checklisten und dem tatsächlichen «Fall» um? Da sind doch auch sie ganz allein gelassen. Auch Reha-Mediziner müssen psychologisch geschult werden. Wir brauchen ein neues Bewusstsein, das weniger auf Normierung und irgendwelche Massstäbe setzt, die am Leben einfach vorbeigehen. Eigentlich gilt das für die ganze Gesellschaft.

Und etwas anderes habe ich deutlich vor Augen geführt bekommen: Es ist etwas ganz tief in uns, das uns stärkt, aber auch die Beziehungen zu anderen Menschen, zur Familie, zu Freunden. Es gibt so viel Erfrischendes und Überraschendes in diesem Buch: zum Beispiel das Einfach-ruhig-Daliegen, mit möglichst wenig Atmung. Das Sich-Leichtmachen in der absoluten Ruhestellung. Leichtigkeit in der Schwere. Und so gibt es so einiges in dem Buch, was mich begeistert hat, auch der Humor.

Das Buch sollte von allen gelesen werden, die mit Rehabilitation zu tun haben: Patienten, Angehörige, Fachpersonen, Sachbearbeiter, Juristen und Politiker. 